\title{
CONHECIMENTO DE PROFISSIONAIS DE ENFERMAGEM ACERCA DA ASSISTÊNCIA À SAÚDE DOS TRANSEXUAIS
}

\author{
Maria Clara Lustosa Fernandes I \\ Willames da Silvall \\ Taysa de Sousa Tolentino III \\ Marilia Juliane Albuquerque Araújo \\ Mayara Layane de Souza Joventino \\ Paulo Emanuel Silva
}

\section{RESUMO}

O tema transexualidade tem adquirido cada vez mais visibilidade. Mas, infelizmente, os serviços de saúde ainda enfrentam uma resistência, não apenas devido ao preconceito, a transfobia, como também por dificuldades de compreensão do princípio da equidade, gerando um desafio para uma assistência de qualidade ao transexual. Nesse contexto, o objetivo deste estudo é averiguar o conhecimento dos profissionais de enfermagem acerca da assistência à saúde e políticas públicas relacionadas aos transexuais. Trata-se de uma pesquisa descritivo-exploratória, com abordagem quantitativa, realizada em um hospital particular de João Pessoa/PB. Foram entrevistados, 40 profissionais de enfermagem (10 enfermeiros e 30 técnicos de enfermagem), por meio da aplicação de questionário semiestruturado. Quanto à assistência à saúde dos transexuais, a maioria dos entrevistados afirmaram já ter prestado atendimento a esses pacientes, porém se mostraram inseguros quanto aos procedimentos corretos. A exemplo da obrigatoriedade de encaminhamentos para consulta com especialista pois, 52,5\% desses profissionais não têm esse conhecimento bem esclarecido, assinalando as opções: provavelmente sim; com certeza não; provavelmente não e não sabe. Ressalta-se, de um modo geral, que a maioria dos profissionais entrevistados, demostraram não possuir conhecimento acerca da forma correta de assistência à saúde dessa população específica, considerando que a maioria afirmou não ajudar na marcação de consulta e não prestar atendimento a esses pacientes, no mesmo dia da procura. Além de não apresentarem conhecimentos em relação a existência e/ou como são instituídas as políticas públicas que envolvem os direitos de pacientes transexuais. Diante disso, os postulados que embasam o sistema de saúde no país impõem uma visão aos profissionais da área de saúde que consiga visualizar as peculiaridades inerentes aos transexuais em relação à sua não compatibilidade entre a identidade de gênero e sexo biológico, garantindo assim assistência especializada para este público.

PALAVRAS-CHAVE: Sexualidade. Identidade de gênero. Orientação sexual.

Enfermeira. Graduada pela Faculdade de Enfermagem Nova Esperança - FACENE João Pessoa, Paraíba, Brasil. Autor correspondente: claralustosa29@gmail.com. ORCID ID: 0000-0002-5577-9977

Enfermeiro. Graduado pela Faculdade de Enfermagem Nova Esperança- FACENE- João Pessoa, Paraíba, Brasil. ORCID ID: 0000-0003-4365-8358

Enfermeira. Pós-Graduanda de Urgência e Emergência com UTI pelo Centro de Aperfeiçoamento III Profissional - CEFAPP - João Pessoa, Paraíba, Brasil. ORCID ID: 0000-0002-5577-9977

Enfermeira. Pós-Graduanda de Urgência e Emergência e UTI pela Faculdade Internacional da Paraíba-FPB - IV João Pessoa, Paraíba, Brasil. ORCID ID: 0000-0002-2319-3015

Acadêmica de enfermagem pela Faculdade de Enfermagem Nova Esperança- FACENE- João Pessoa, Paraíba, Brasil. ORCID ID: 0000-0001-5532-5580

Enfermeiro. Mestre em Ciências das Religiões pela Universidade Federal da Paraíba. Docente da IV FACENE, João Pessoa, Paraíba, Brasil. ORCID ID: 0000-0001-9326-4619 


\section{INTRODUÇÃO}

A transexualidade é caracterizada pelo sentimento acentuado de um indivíduo seja homem ou mulher ao não pertencimento ao sexo biológico, buscando se adequar à identidade de gênero. Na maioria dos casos, as pessoas trans recorrem a tratamentos médicos que vão desde a terapia hormonal até a cirurgia de redesignação sexual. ${ }^{1}$

Nesse processo, deve-se incluir a terapia hormonal, o acompanhamento multidisciplinar e a cirurgia de readequação sexual, que deve ser disponibilizada gratuitamente pelo SUS. E cabe a Secretaria de Atenção à Saúde e ao Ministério da Saúde estruturar as secretarias dos estados, dos municípios e do Distrito Federal para a implementação desses atendimentos. As pessoas que passarem pelo processo transexualizador também terão acompanhamento psicológico e atendimento multidisciplinar. ${ }^{2}$

O transexualismo era considerado pela Classificação Internacional de Doenças, décima edição (CID-10), como transtorno mental, caracterizado pela disforia de gênero.3 Posteriormente a revisão da CID-10 e a publicação da quinta edição do Manual Diagnóstico e Estatístico de Transtornos Mentais (DSM) redefiniram a tal classificação como incongruência de gênero, sendo considerada como uma variação da normalidade e não um transtorno sexual, como até então era considerado pelos manuais. 4

O Departamento de Apoio à Gestão Participativa da Secretaria de Gestão Estratégica e Participativa do Ministério da Saúde lançou, através da Portaria $n^{\circ}$ 2.836/11 a Política Nacional de Saúde Integral da população LGBTs, dando ênfase na sua equidade e integralidade e na qualificação dos profissionais de saúde para o trabalho humanizado e livre de preconceitos. 5 Torna-se importante ressaltar que os trabalhadores da área da saúde, ao se depararem com um indivíduo transexual nos serviços
VOLUME17-NÚMERO2AGO/2019
ISSNELETRÔNICO2317-7160

de saúde, devem pautar-se com respeito, sem preconceito e discriminação visando proporcionar seu bem-estar biopsicossocial, independentemente de sua identidade de gênero. ${ }^{6}$

O Art. $3^{\circ}$, inciso IV da Constituição Federal de 1988, afirma que os cidadãos não podem sofrer nenhum tipo de preconceito, sendo necessário promover ações de conscientização e aceitação da diversidade humana principalmente as que estão relacionadas ao público LGBT.7 A assistência de enfermagem à população transexual consiste na prevenção, promoção e proteção do processo saúde-doença deste público, visando à garantia de uma assistência digna e humanizada, desde a atenção básica de saúde, até a média e alta complexidade, proporcionando ações fundamentais para promoção integral de saúde de forma efetiva e de qualidade. ${ }^{8}$

Diante deste contexto, o nome social passa a ser incluído nos prontuários médicos em março de 2006, reconhecido pela portaria $\mathrm{N}^{\circ} 675 / \mathrm{GM}$, na tentativa de tornar $\mathrm{o}$ atendimento igualitário, independentemente da idade, etnia, orientação sexual ou de identidade de gênero, garantindo assim, a equidade, integralidade, universalidade em estratégias de prevenção de doença, promoção à saúde, sem preconceito ou discriminação relacionado aos grupos sociais. 9

Sendo assim, os profissionais de saúde devem propiciar um ambiente acolhedor, a partir de reflexões sobre as crenças, preocupações, orientaçõese tabus sobre a sexualidade. O enfermeiro oferece um atendimento humanizado livre de qualquer preconceito e discriminação, assegurando o uso do nome social no caso dos transexuais como uma estratégia de promoção e acesso ao sistema de saúde. ${ }^{10}$ Portanto, o presente estudo objetiva averiguar o conhecimento dos profissionais de enfermagem acerca da assistência à saúde dos transexuais. 


\section{MATERIAL E MÉTODOS}

Este estudo se caracteriza como uma pesquisa de caráter descritiva com abordagem quantitativa, realizada em um hospital particular, localizado no município de João Pessoa- PB, realizada no ano de 2018, adotando as seguintes etapas:

Etapa 1 - Consistiu de um contato prévio com profissionais de enfermagem do HUNE, convidados a participar da pesquisa, quando foram explanados os objetivos e justificativas do estudo.

Etapa 2 - Foi explicado a importância de sua participação e apresentação do Termo de Consentimento Livre Esclarecido, dando garantia do seu anonimato.

Etapa 3 - Foi aplicado como instrumento para coleta de dados, questionário semiestruturado para autopreenchimento, conforme Tabela 1.

Tabela 1. Questionário detalhado utilizado para entrevista realizada com os profissionais de enfermagem de um hospital particular. João Pessoa -PB

\begin{tabular}{|c|c|}
\hline \multicolumn{2}{|c|}{ CARACTERIZAÇÃO PROFISSIONAL DOS ENTREVISTADOS } \\
\hline Marque uma das alternativas & $\begin{array}{l}\text { Enfermeiro } \\
\text { Téc. Enfermagem }\end{array}$ \\
\hline \multicolumn{2}{|c|}{ CONHECIMENTO DOS PROFISSIONAIS ACERCA DO TRANSEXUALISMO } \\
\hline $\begin{array}{l}\text { Você considera uma pessoa transexual (es- } \\
\text { colher apenas uma resposta): }\end{array}$ & $\begin{array}{l}\text { Homem que se veste como } \\
\text { hulher/mulher que se veste com } \\
\text { omem } \\
\text { )Homem parecido com mul- } \\
\text { er/mulher que se parece com } \\
\text { omem } \\
\text { Homem ou mulher que tenta se } \\
\text { proximar do corpo que deseja } \\
\text { feminino ou masculino) }\end{array}$ \\
\hline \multicolumn{2}{|c|}{ ASSISTÊNCIA Ȧ SAÚDE PRESTADA AOS TRANSEXUAIS } \\
\hline $\begin{array}{l}\text { Já foram atendidos transexuais neste } \\
\text { hospital? }\end{array}$ & $\begin{array}{l}\text { Sim } \\
\text { Não }\end{array}$ \\
\hline $\begin{array}{l}\text { Quando um paciente transexual tem que } \\
\text { consultar um especialista, o serviço de saúde/ } \\
\text { ou médico/enfermeiro faz encaminhamentos } \\
\text { obrigatoriamente? }\end{array}$ & $\begin{array}{l}\text { Com certeza, sim } \\
\text { Provavelmente, sim } \\
\text { Provavelmente, não } \\
\text { Com certeza, não } \\
\text { Não sei }\end{array}$ \\
\hline $\begin{array}{l}\text { Alguém que trabalha neste serviço de saúde } \\
\text { ajuda o usuário a marcar consultas com um }\end{array}$ & $\begin{array}{l}\text { Com certeza, sim } \\
\text { Provavelmente, sim } \\
\text { Provavelmente, não } \\
\text { Com certeza, não } \\
\text { Não sei }\end{array}$ \\
\hline $\begin{array}{l}\text { Quando o usuário transexual adoece, } \\
\text { alguém do serviço faz o atendimento no } \\
\text { mesmo dia? }\end{array}$ & $\begin{array}{l}\text { Com certeza, sim } \\
\text { Provavelmente, sim } \\
\text { Provavelmente, não } \\
\text { Com certeza, não } \\
\text { Não sei }\end{array}$ \\
\hline \multicolumn{2}{|c|}{ CONHECIMENTO DAS POLÍTICAS PÚBLICAS VOLTADAS PARA POPULAÇÃO LGBT } \\
\hline $\begin{array}{l}\text { Tem conhecimento da existência de políticas } \\
\text { públicas de saúde relacionada a população } \\
\text { LGBT, em que se inclui os transexuais? }\end{array}$ & $\begin{array}{l}\text { ) Sim } \\
\text { ) Não }\end{array}$ \\
\hline $\begin{array}{c}\text { Sabe informar qual portaria instituída pelo } \\
\text { Ministério da Saúde orienta ações integrais a } \\
\text { essa população? }\end{array}$ & $\begin{array}{l}\text { ) Sim } \\
\text { Não }\end{array}$ \\
\hline
\end{tabular}


O questionário foi preenchido por 10 enfermeiros e 30 técnicos de enfermagem, somando 40 profissionais de enfermagem participantes da pesquisa. As respostas obtidas para cada ponto do questionário foram transformadas em percentuais e representa- das na forma de gráficos e tabelas.

Foi aprovado pelo Comitê de Ética em Pesquisa da Faculdade de Enfermagem e Medicina Nova Esperança - FACENE/FAMENE, sob o Protocolo de $n^{\circ}$ 125/2017 e CAAE: 75007017.4.0000.5179.

\section{RESULTADOS E DISCUSSÃO}

Na Figura 1, consta a distribuição da amostra, de acordo com a categoria profissional que respondeu o questionário, em que $25 \%$ dos entrevistados são enfermeiros e $75 \%$ técnicos de enfermagem. Percebe-se que há predominância dos técnicos de enfermagem. Essa divisão é realizada através do dimensionamen- to do pessoal de enfermagem, que tem como objetivo a distribuição do percentual do total de profissionais de enfermagem, considerando a proporção profissional/paciente e estabelecendo o quadro quantitativo de profissionais necessários para a prestação da assistência de enfermagem aos pacientes.

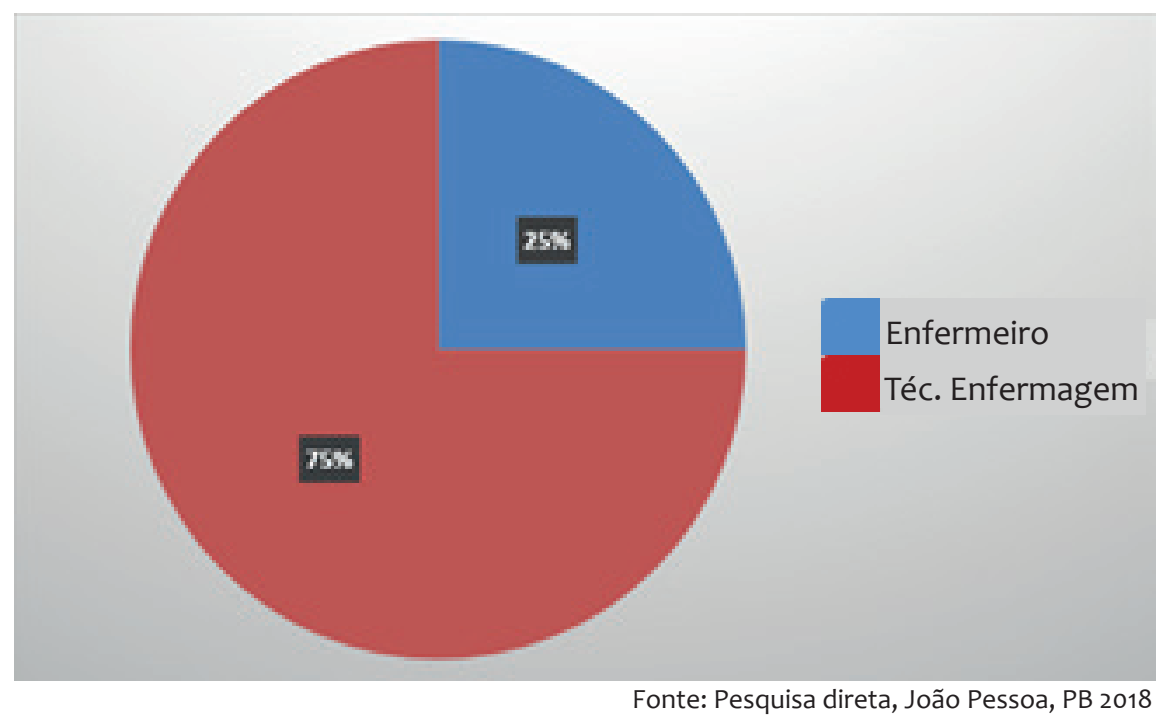

FIGURA 1- Amostragem dos profissionais de enfermagem de um hospital particular, de acordo com a categoria, que aceitaram participar do estudo acerca do conhecimento da assistência à saúde dos transexuais

Para se determinar a distribuição do pessoal de enfermagem é necessário seguir os critérios, de acordo com o COFEN, através da resolução 543/2017 que diz: 
Para cuidado mínimo e intermediário: 33\% são enfermeiros (mínimo de seis) e os demais auxiliares e/ou técnicos de enfermagem; para cuidado de alta dependência: $36 \%$ são enfermeiros e os demais técnicos e/ou auxiliares de enfermagem; para cuidado semi-intensivo: $42 \%$ são enfermeiros e os demais técnicos de enfermagem; para cuidado intensivo: $52 \%$ são enfermeiros e os demais técnicos de enfermagem. ${ }^{11}$

Inicialmente, os profissionais foram arguidos quanto ao conhecimento sobre o transexualismo. Neste sentido, observa-se na Figura 2 que a maioria dos participantes deste estudo têm conhecimento sobre a Transexualidade, em detrimento de $12,5 \%$ que demos- traram não saberem o significado do termo. Diante disto, vale ressaltar que toda a equipe de profissionais da gestão, desde as unidades básicas aos hospitais, deve ser informada e sensibilizada sobre o acolhimento e o atendimento holístico as pessoas LGBT.

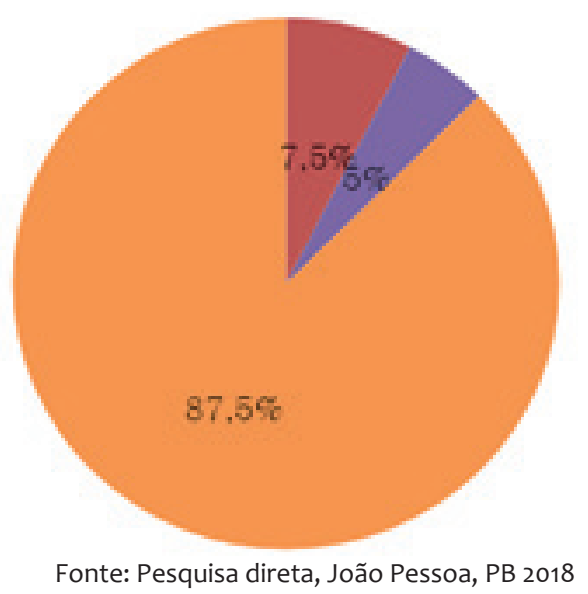

Homem que se veste como mulher/mulher que se veste com homem

Homem parecido com mulher/mulher que se parece com homem

Homem ou mulher que tenta se aproximar do corpo que deseja (feminino ou masculino)

FIGURA 2- Nível de conhecimento, dos profissionais de enfermagem de um hospital particular $(\mathrm{n}=40)$, acerca do transexualismo

Esses resultados reforçam que, mesmo com o trânsito mais assíduo, devido aos movimentos organizados LGBT, a realidade dessa população ainda passa por conflitos, seja para ela que precisa dos serviços, seja para os profissionais que ainda não compreendem as especificidades de alguns grupos populacionais. ${ }^{12}$

Os transexuais são indivíduos que nascem em um sexo, mas que se identificam como membro do sexo oposto. Por isso, tomam hormônios e submetem-se a intervenções cirúrgicas para remodelar a genitália. $\mathrm{O}$ transexua- lismo, por seu turno, ocorre quando a pessoa rejeita sua própria identidade sexual e se identifica psicologicamente com o gênero oposto, havendo uma confusão entre a identidade sexual física (o que a pessoa é) com a identidade sexual psíquica (o que ela pensa ser), ocasionando, através de uma neurose racional obsessivo-compulsiva, o desejo de reversão sexual integral. ${ }^{13}$

A assistência aos transexuais deve sempre seguir os preceitos da universalidade, integralidade e equidade da atenção, 
princípios resguardados pelo SUS. Infelizmente, os serviços de saúde enfrentam resistências, devido não apenas ao preconceito, a transfobia e a discriminação, incutidas em algumas práticas de saúde, como também dificuldades de compreensão da importância do princípio da equidade. $O$ grande desafio para uma assistência de qualidade ao transexual é o reconhecimento do sofrimento psíquico e a importância de um atendimento humanizado com o olhar holístico. 6

Na Tabela 2, observa-se um perfil da assistência, prestada pelos profissionais de enfermagem, a pacientes transexuais ao procurar atendimento. É possível observar que a maioria dos entrevistados (67,5\%) já atenderam pacientes transexuais no referido hospital. Porém, apresentam-se inseguros quanto a devida forma de assistência, considerando que a maioria $(52,5 \%)$ não tem certeza da obrigatoriedade do encaminhamento dos pacientes transexuais para consulta com especialista.

Ressalta-se que os demais questionamentos demonstram que apenas $30 \%$ dos entrevistados ajudam na marcação de consultas e $55 \%$ realizam o atendimento no mesmo dia.

TABELA 2- Perfil dos profissionais de enfermagem de um hospital particular de João Pessoa $(n=40)$, em relação a assistência prestada aos transexuais

\begin{tabular}{lcc}
\hline Questionamentos & $\mathrm{N}$ & $(\%$ \\
\hline \hline Já atenderam transexuais? & 13 & 32,5 \\
\hline \hline ( ) Sim & 27 & 67,5 \\
( ) Não & 19 & 47,5 \\
\hline \hline Obrigatoriedade de encaminhamento para consulta com especialista & \\
\hline \hline ( ) Com certeza, sim & 8 & 20 \\
( ) Provavelmente, sim & 6 & 15 \\
( ) Com certeza, não & 1 & 2,5 \\
( ) Provavelmente, não & 6 & 15 \\
( ) Não sei & 12 & 30 \\
\hline \hline Obrigatoriedade de encaminhamento para consulta com especialista & \\
\hline \hline ( ) Com certeza, sim & 11 & 27,5 \\
( ) Provavelmente, sim & 1 & 2,5 \\
( ) Com certeza, não & 16 & 40 \\
( ) Não sei & Fonte: Pesquisa direta, João Pessoa, PB 2018
\end{tabular}




\section{revista de NOVA \\ da saúde ESPERANÇA}

No contexto da saúde, de um modo geral, espera-se do profissional de Enfermagem uma visão ampla e humanitária sobre o Sistema Único de Saúde (SUS), no qual, no art. 196 da Constituição Federal (CF) de 1988, versa, como um dos princípios, a universalidade, que garante a atenção à saúde a todo cidadão "Saúde é um direito de todos e dever do estado". Bem como, vivenciando a prática de atendimento é de se esperar, com os princípios da integralidade e equidade da mesma constituição, que o sistema de saúde esteja preparado e capacitado para atender todo e qualquer tipo de usuário que necessite do serviço ali prestado, levando em consideração o contexto social de cada usuário. ${ }^{14}$

A linha de cuidado da atenção a transexuais é estruturada por dois pilares: o primeiro é a atenção básica e a segunda é a atenção especializada (ambulatorial e hospitalar). A atenção básica é a porta de entrada do SUS, ou seja, é o primeiro serviço que o usuário pode fazer uso, e deve garantir o cuidado integral, através do acolhimento humanizado, do uso do nome social do usuário e do encaminhamento adequado ao Serviço de Atenção Especializada. Já a atenção especializada é considerada como um conjunto de diversos pontos de atenção, com diferentes densidades tecnológicas para a realização de ações e serviços de urgência, ambulatorial especializado e hospitalar, apoiando e complementando os serviços da atenção básica de forma resolutiva e em tempo oportuno. ${ }^{15}$

Atualmente, no Brasil, existem 10 serviços especializados em transexualidade. Destes, apenas 05 estão credenciados ao SUS com equipes formadas por médicos urologistas, endocrinologistas, ginecologistas, cirurgiões, psiquiatras, psicólogos e assistentes sociais. Ressalta-se que, apenas na Universidade do Estado do Rio de Janeiro (UERJ) e no Hospital de Clínicas de Goiânia, há enfermeiros inseridos na equipe. ${ }^{16}$
No Brasil, foi instituída, no âmbito do SUS, a Política Nacional de Saúde Integral de Lésbicas, Gays, Bissexuais, Travestis e Transexuais (PNSI-LGBT), através da Portaria GM/MS $\mathrm{n}^{\circ} 2.836$, de $1^{\circ}$ de dezembro de 2011, ficando a cargo das secretarias estaduais a definição das estratégias e do plano de ação para sua implementação e das secretarias municipais sua efetivação, a partir da identificação das necessidades de saúde da população LGBT em cada município. ${ }^{17}$

Apesar das conquistas obtidas, não podemos deixar de lembrar que a população transexual, infelizmente, ainda é bastante recriminada e colocada à margem da sociedade. Com isso, o transexual carrega em si o sentimento de inferioridade em relação ao resto da sociedade, que o acaba prejudicando psicologicamente.

Os problemas enfrentados pela população LGBT, acerca do acesso a serviços de saúde, são ainda mais dramáticos nos casos de travestis e transexuais. Não só por reivindicarem atendimento especializado para demandas que não se colocam para outros segmentos populacionais (alterações corporais associadas ao uso de hormônios e silicone, por exemplo), mas também pela intensidade da transfobia que costuma incidir sobre estes dois grupos indenitários. Também pela intensidade da transfobia que costuma incidir sobre estes dois grupos indenitários. Provavelmente, devido esses apresentarem-se como o público, que mais intensamente sente a escassez de políticas de saúde específicas e, paralelamente, o que mais se beneficiará de tais ações quando forem efetivadas. ${ }^{16}$

As desigualdades de acesso encontram-se como um dos principais problemas a serem enfrentados para que o SUS possa funcionar conforme seus princípios e diretrizes. E para que o acesso na Atenção Primária a Saúde seja equitativo, garantindo a entrada de pessoas transexuais faz-se necessário ampliar 
o debate sobre diversidade sexual e de gênero. Políticas de saúde devem ser capazes de eliminar as barreiras geográficas, econômicas e culturais entre os serviços e a comunidade, além de garantir que as pessoas trans permaneçam sob o cuidado integral de profissionais de saúde. ${ }^{18}$

Em razão disso, no que diz respeito à saúde da população transexual, é indispensável um tratamento especializado que advém das particularidades que envolvem os corpos e as mentes dos transexuais. É comum a necessidade de atendimento médico, devido a automutilação do órgão genital, de complicações derivadas de procedimentos de modificação dos órgãos sexuais secundários (implantes caseiros) e de adversidade ocasionadas pela ingestão excessiva de hormônios. Somente por meio do reconhecimento da diferença é que se torna possível a efetivação do direito à saúde desse grupo tão vulnerável. ${ }^{19}$

A Tabela 3 elenca os participantes do estudo, de acordo com conhecimento das políticas públicas relacionadas a população LGBT, na qual se percebe que um maior percentual afirma conhecer essas políticas, totalizando $60 \%$ dos entrevistados. A portaria instituída pelo Ministério da Saúde orienta ações integrais a essa população, mas $70 \%$ (28) dos entrevistados afirmaram não ter conhecimento.

TABELA 3: Perfil dos profissionais de enfermagem de um hospital particular de João Pessoa $(n=40)$, relacionado ao conhecimento das políticas públicas voltadas para a população LGBT

\begin{tabular}{lcc}
\hline \hline \multicolumn{1}{c}{ Variável } & $\mathrm{N}$ & $(\%)$ \\
\hline \hline Conhecimento das políticas públicas voltadas para a população LGBT & & \\
\hline \hline ( ) Sim & 16 & 40 \\
( ) Não & 60 & 60 \\
\hline \hline Sabe informar qual portaria instituída pelo Ministério da Saúde orienta ações inte- \\
grais a essa população? & 12 & 30 \\
\hline ( ) Sim & 28 & 70 \\
( ) Não & Fonte: Pesquisa direta, João Pessoa, PB.
\end{tabular}

'Lésbicas, Gays, Bissexuais, Travestis, Transexuais e Transgêneros

Neste contexto, ao tratar das relações entre discriminação e saúde, afirmam que a discriminação constitui uma questão relevante com a qual profissionais, gestores e acadêmicos da área da saúde devem lidar. Os autores apontam que há uma vasta literatura que documenta a influência deletéria de processos discriminatórios na relação estabelecida entre profissionais da saúde e pacientes, na prescrição de medicamentosos ou de outros procedimentos cirúrgicos e terapêuticos, assim como na própria satisfação dos usuários com o atendimento prestado. ${ }^{16,20}$

Corrobora com essa afirmação e acrescentam que operando de forma excludente e preconceituosa, o sistema de saúde informa, mesmo que implicitamente, quando o usuário é ou não bem-vindo, não sendo o usuário quem define se vai acessar ou não o sistema; é o sistema que diz quem, quando, onde e de que forma se dará o atendimento. ${ }^{16}$

As demandas do movimento LGBT, 
quando inseridas em políticas públicas, apresentam como primeiro marco, o Programa Nacional de Direitos Humanos (PNDH), o qual abordava cinco ações que contemplavam a orientação sexual, enquanto dimensão da garantia do direito à liberdade, opinião e expressão e outras dez relacionadas a garantia do direito à igualdade de gays, lésbicas, bissexuais, travestis e transexuais. ${ }^{21}$

Torna-se importante enfatizar que a população LGBT, em função de sua distinção heteronormativa, passa por situações de cerceamento dos direitos humanos básicos que podem repercutir para uma situação de vulne-

\section{CONSIDERAÇÕES FINAIS}

A partir dessa reflexão, conclui-se que a maioria dos profissionais de saúde têm algum conhecimento sobre a assistência à saúde dos transexuais. Porém, se faz necessária uma adequação das práticas em saúde, ofertadas pelos serviços, com atuação de profissionais qualificados e informados sobre a cultura heteronormativa, buscando garantir o acesso integral e o cuidado à saúde sem preconceito e discriminação.

Para lidar convenientemente com os aspectos da sexualidade, dentro do serviço assistencial, é essencial a adequação da abordagem aos usuários sobre seu estilo de vida, respeitando e abrangendo a atenção, capaz de utilizar técnicas e habilidades que promovam a verdadeira resolutividade as necessidades apontadas por esses pacientes. Para isto, é preciso que os profissionais da equipe multiprofissional estejam habilitados no acolhimen- rabilidade. ${ }^{22}$ Além disso, a identidade sexual e a identidade de gênero são representações de um processo complexo de discriminação e de exclusão que pode levar a fatores de vulnerabilidade, a exemplo da violação do direito à saúde, à dignidade, à autonomia e ao livre desenvolvimento. 23

Nesse contexto, o gênero

é uma das perspectivas das relações sociais que produz desigualdades. Então a política de saúde, desenvolvida no âmbito do SUS, deve reconhecer a existência dessas desigualdades e respondê-las, com vistas a promoção da equidade de gênero. 24

to e que o cuidado seja centrado na pessoa que está diante deles em um evento marcado por estigmas e medos.

Por fim, os postulados que embasam o sistema de saúde no país (universalidade, integralidade e equidade) impõem uma visão aos profissionais da área de saúde que consiga visualizar as peculiaridades inerentes aos transexuais em relação à sua não compatibilidade entre a identidade de gênero e sexo biológico, garantindo assim assistência especializada para este público, visto que a saúde do transexual não se resume à cirurgia de redesignação sexual.

Espera-se que esse trabalho tenha sido de significativa contribuição e acréscimo à literatura já existente, servindo de referência para outros estudos que abordem essa temática. 


\title{
KNOWLEDGE OF NURSING PROFESSIONALS ABOUT THE TRANSEXUAL HEALTH CARE ASSISTANCE
}

\begin{abstract}
The issue of transsexuality has become increasingly important, but unfortunately, the health services still face resistance, not only due to prejudice, transphobia, but also because of difficulties in understanding the principle of equity, creating a challenge for quality assistance to the transsexual. In this context, the objective of this study is to ascertain the knowledge of nursing professionals about health care and public policies related to transsexuals. This is a descriptive-exploratory study, with a quantitative approach, performed in a private hospital, in João Pessoa / PB. We interviewed 40 nursing professionals (10 nurses and 30 nursing technicians), through the application of a semi-structured questionnaire. Regarding health care for transsexuals, most of the interviewees affirmed that they had already provided care to these patients, but were unsure about the correct procedures. As an example of the requirement of referrals for consultation with a specialist, where $52.5 \%$ of these professionals do not have this knowledge well informed, pointing out the options: probably yes; certainly not; probably not and do not know. It should be noted, in general, that the majority of the professionals interviewed, showed that they did not have knowledge about the correct form of health care for this specific population, considering that the majority affirmed, did not help to set the appointment and did not provide care on the same day. In addition, they do not have knowledge about the existence and / or how the public policies are formed and which involve the rights of transsexual patients. Therefore, the postulates that underlie the health system in the country impose a vision for health professionals who can visualize the peculiarities inherent to transsexuals in relation to their non-compatibility between gender identity and biological sex, thus guaranteeing specialized assistance to this public.
\end{abstract}

KEYWORDS: Sexuality. Gender identity. Sexual Orientation.

\section{REFERÊNCIAS}

1. Galli RA, Vieira EM, Giami A, Santos MA. Corpos Mutantes, Mulheres Intrigantes: Transexualidade e Cirurgia de Redesignação Sexual. Psicologia: Teoria e Pesquisa. Out./dez. 2013; 29(04): 447-57.

2. Araújo IA, Tavares ALB, Souza ACC, Rodrigues FJS. Pessoas transexuais e o acesso aos serviços de saúde no Brasil: revisão integrativa. Cadernos Esp. Ceará. Jul./dez. 2018; 12(2): 112-27.

3. Moreira JAC, Padilha MI. Trans-formação: uma revisão sobre os principais conceitos da transexualidade. Eletrônica Estácio Saúde. 2015; 4(1): 34-44.
4. Spizzirri G. Disforia de gênero em indivíduos transexuais adultos: aspectos clínicos e epidemiológicos. 2017; 22(01):45-8.

5. Rogers J, Tesser-Júnior ZC, Pires ROM, Kovaleski DF. Pessoas Trans na Atenção Primária: análise preliminar da implantação no município de Florianópolis, 2015. Sau. \& Transf. Soc. 2016; 7(3): 49-53.

6. Maksoud FR, Passos XS, Pegoraro RB. Reflexões acerca do transtorno de identidade de gênero frente aos serviços de saúde: revisão bibliográfica. Rev. Psicol. Saúde. 2014; 6(2): 47-55. 
7. Querino MS, Almeida SS, Oliveira SCS, Umann J, Moraes-Filho IM. Ações da equipe de enfermagem na implementação da política de lésbicas, gays, bissexuais, travestis e transexuais- revisão de literatura. Rev. Cient. Sena Aires. 2017; 6(1): 46-58.

8. Silva GWS, Sena RCF, Santos QG, Sobreira MVS, Miranda FAN. O dito e o feito: o enfermeiro e o saber/fazer saúde para travestis. Rev. Enferm. UFPE on line. Out. 2014; 8(10): 3347-57.

9. Pereira LBC, Chazan ACS. O Acesso das Pessoas Transexuais e Travestis à Atenção

Primária à Saúde: uma revisão integrativa. Rev. Bras. Med. Fam. Comunidade. 2019; 14(41): 1795.

10. Braga LS, Martins BG, Oliveira ARC, Rodrigues RF, Soier RMS, Souza SO, Resende FA et al. Inovação da assistência à saúde prestada à população lésbica, bissexuais, gays, travestis e transexuais. Rev. Bras. Ciências da Vida. Abr. 2018; 6(especial).

11. Conselho Federal de Enfermagem (BR). Resolução 543/2017: Dimensionamento do Quadro de Profissionais de Enfermagem nos serviços/locais em que são realizadas atividades de enfermagem. Brasília: COFEN; 2017.

12 Ministério da Saúde (BR). Relatório Gestão 2015. Brasília: Ministério da Saúde; 2015.

13. Carrieri AP, Souza EM, Aguiar ARC. Trabalho, Violência e Sexualidade: Estudo de Lésbicas, Travestis e Transexuais. Revista de Administração Contemporânea. Jan./fev. 2014; 15(1): 78-95.

14. Campelo RR, Peres EM. Analise do atendimento humanizado no SUS aos Transexuais. Rev. Cient. do Instituto Ideia. 2018; 1(7): 83-96.

15. Preu RO, Brito CF. A questão trans no cenário brasileiro. Rev. Est. Ind. Gêneros e Sexualidade. 2018; 1(10): 95-117.
16. Guaranha C. Travestis e transexuais: a questão da busca pelo acesso à saúde. Seminário Internacional Fazendo Gênero. Florianópolis: 2013. 17 Gomes SM, Sousa LMP, Vasconcelos TM, Nagashima AMS. O SUS fora do armário: concepções de gestores municipais de saúde sobre a população LGBT. Rev. Sau. Soc. 2018; 27(4): 112033.

18. Pereira LBC, Chazan ACS. O Acesso das Pessoas Transexuais e Travestis à Atenção Primária à Saúde: uma revisão integrativa. Rev. Bras. Med. Fam. Comunidade. 2019; 14(41): 1795.

19. Gomes LGC, Lima JN. Horizontalidade social trans: o direito social à saúde dos transexuais nas relações entre particulares. Rev. Jur. Cesumar. Jan./abr. 2019; 19(1): 39-63.

20. Bastos JL, Faerstein E. Desafios metodológicos para a mensuração da discriminação interpessoal no Brasil. Discriminação e saúde: perspectivas e métodos. Rio de Janeiro: Editora Fiocruz, 2012. p. 75-102.

21. Mello L, Brito W, Maroja D. Políticas públicas para a população LGBT no Brasil: notas sobre alcances e possibilidades. Cad. Pagu. Jul./dez. 2012; 39: 403-29.

22. Cardoso MR, Ferro LF. Saúde e População LGBT: Demandas e Especificidades em Questão. Psicol. Ciênc. Prof. Fev. 2012; 32(3): 552-63.

23. Ministério da Saúde (BR). Saúde da população de gays, lésbicas, bissexuais, travestis e transexuais. Rev. Sau. Pública, Brasília: Ministério da Saúde; 2016.

24 Ferraz D, Kraiczyk J. Gênero e Políticas Públicas de Saúde - construindo respostas para o enfrentamento das desigualdades no âmbito do SUS. Rev. Psic. UNESP. 2010; 9(1): 70. 